\title{
Um estudo da propagação geográfica da dengue no Peru
}

\author{
Nelson Q. Cuba ${ }^{1}$ \\ IMECC/UNICAMP, Campinas, SP \\ Lucy T. Takahashi ${ }^{2}$ \\ ICE/UFJF, Juiz de Fora, MG
}

\begin{abstract}
Resumo. A dengue é uma doença causada por um arbovírus e seu principal vetor é o mosquito $A e$ des aegypti, que a cada ano invade novas regiões tornando-se endêmica. Neste trabalho as dinâmicas da dengue e do mosquito Aedes aegypti são descritas, espaço-temporal, por um modelo de metapopulação, para se determinar um possível caminho que a doença percorreu para chegar até a região norte de Ayacucho, estado no interior do Peru. Para descrever essa propagação considera-se uma rede formada de $n$ cidades, que estão conectadas entre si, por rodovias ou por hidrovias. Em cada uma das $n$ cidades considera-se uma dinâmica $S I$ para a população de mosquitos com migração e uma dinâmica $S I R$ para a população humana com residência fixa. Além de estudar o modelo na sua forma adimensional, determinamos o número de reprodutibilidade basal, $\mathcal{R}_{0}$, por meio da matriz da próxima geração. E os resultados das simulações para descrever essa propagação são compatíveis com os dados históricos levantados. Além disso, o problema foi implementado computacionalmente no software GNU Octave com códigos que usam o pacote ode23.
\end{abstract}

Palavras-chave. Epidemiologia, Ayacucho, Dispersão, Rodovias, EDO.

\section{Introdução}

Segundo o Ministério da Saúde do Peru (MINSA), os primeiros casos de dengue no Peru eram do sorotipo DEN-1 e foram registrados em 1990 na cidade de Iquitos, na região norte da Amazônia peruana. E nos próximos anos a dengue espalhou-se por mais cidades da região centro e norte da Amazônia peruana até chegar a região norte do Litoral peruano e provocar uma epidemia em 2000. A dengue continuou a espalhar-se em direção ao sul do Litoral chegando a capital, Lima, em 2005. O segundo sorotipo a ingressar no Peru foi o DEN-2 em 1995 também sucedeu na cidade de Iquitos [1]. A partir do ingresso dos sorotipos DEN-3 e DEN-4 na cidade de Piura na região norte do Litoral começa a primeira epidemia da dengue hemorrágica na mesma região, a segunda epidemia da dengue hemorrágica foi entre 2010 e 2011 ocasionada pelo sorotipo DEN-2 já inciando-se na cidade de Iquitos e espalhando-se para as cidades da Amazônia.

Por outro lado, de acordo com o MINSA, na região sul da Amazônia peruana a partir de 2000 a dengue permaneceu localizada numa parte do estado do Madre de Dios, o qual faz divisa com o estado do Acre no Brasil. Mas, desde a construção da Rodovia Interoceânica em 2007 aumentou o fluxo de veículos e assim a dengue foi espalhando-se para as cidades vizinhas. Deste modo, a dengue continuou espalhando-se e chegou a região norte de Ayacucho, que fica no interior do Peru, em 2013, e somente a partir de 2015 Ayacucho reporta casos da dengue a cada ano.

Nesse trabalho, determinamos possíveis caminhos que a dengue deve ter percorrido até chegar a região norte do estado de Ayacucho, no Peru, e entre estes possíveis o que mais se ajusta aos dados históricos da doença no país.

\footnotetext{
${ }^{1}$ nelcuba.mat@gmail.com.

2ltiemi@gmail.com.
} 


\section{O Modelo}

Takahashi et al. (2004), [4], propuseram um modelo para descrever a propagação da dengue através do estado de São Paulo, no Brasil, o qual considera uma rede de $n$ cidades e o fluxo de veículos entre algumas dessas cidades. No modelo, a dinâmica da população de mosquitos infectado pela dengue migra entre as cidades conectadas, e a dinâmica da população humana é sem migração, mas podendo realizar visitas às cidades conectadas a sua. Neste trabalho, propomos mudanças ao modelo de Takahashi et al. (2004), a mudança consiste em considerar além da migração dos mosquitos infectados a migração dos mosquitos sadios, pois de acordo aos dados levantados da propagação da dengue no Peru, o mosquito e a dengue aparentemente foram se espalhando simultaneamente pelo território peruano. Também consideramos a conexão das cidades por meio do fluxo fluvial.

Consideramos para a propagação geográfica da dengue compartimentos do tipo SIR para os humanos, $\left(H_{S}^{i}, H_{I}^{i}, H_{R}^{i}\right)$, e do tipo $S I$ para os mosquitos, $\left(M_{S}^{i}, M_{I}^{i}\right)$ em cada cidade, $i$, com $i=$ $1, \ldots, n$, onde $n$ é o número de cidades da rede. A população de humanos é considerada constante em cada cidade, $i$. isto é, tomamos $H^{i}=H_{S}^{i}+H_{I}^{i}+H_{R}^{i}$ constante, então determinando $H_{S}^{i}$ e $H_{I}^{i}$ podemos achar o $H_{R}^{i}$, pois o $H^{i}$ já é conhecida. Desse modo, considerando também as modificações mencionadas no modelo de Takahashi et al. (2004), propomos o modelo

$$
\left\{\begin{array}{l}
\frac{d M_{S}^{i}}{d t}=\nu^{i} M^{i}\left(1-\frac{M^{i}}{k^{i}}\right)-\mu_{M}^{i} M_{S}^{i}-\beta^{i} M_{S}^{i} H_{I}^{i}-\sum_{j \neq i} f_{j i} \beta^{i} M_{S}^{i} H_{I}^{j}+\sum_{j=1}^{n} \tau_{j i} M_{S}^{j} \\
\frac{d M_{I}^{i}}{d t}=-\mu_{M}^{i} M_{I}^{i}+\beta^{i} M_{S}^{i} H_{I}^{i}+\sum_{j \neq i} f_{j i} \beta^{i} M_{S}^{i} H_{I}^{j}+\sum_{j=1}^{n} \tau_{j i} M_{I}^{j} \\
\frac{d H_{S}^{i}}{d t}=\mu_{H}\left(H^{i}-H_{S}^{i}\right)-\lambda^{i} H_{S}^{i} M_{I}^{i}-\sum_{j \neq i} f_{i j} \lambda^{j} H_{S}^{i} M_{I}^{j} \\
\frac{d H_{I}^{i}}{d t}=-\left(\mu_{H}+\alpha\right) H_{I}^{i}+\lambda^{i} H_{S}^{i} M_{I}^{i}+\sum_{j \neq i} f_{i j} \lambda^{j} H_{S}^{i} M_{I}^{j} .
\end{array}\right.
$$

onde $k^{i}$ é a capacidade de suporte e $\nu^{i}, \beta^{i}, \mu_{M}^{i}$ são as taxas de oviposição, de contágio e de mortalidade dos mosquitos, respectivamente; e $\lambda^{i}, \alpha^{i}, \mu_{H}$ são as taxas de contágio, de recuperação e de mortalidade dos humanos, respectivamente; $\tau_{i j}$ representa a taxa de migração dos mosquitos da cidade $i$ para a cidade $j$ e $f_{i j}$ representa as taxa de transporte dos humanos da cidade $i$ para a cidade $j$.

\subsection{Análise qualitativa do modelo sem migração}

Afim de realizar uma análise qualitativa e assim determinar o número básico de reprodutibilidade basal, $\mathcal{R}_{0}$, consideramos as $n$ cidades como uma só, logo tomamos $H=\sum_{i=1}^{n} H^{i}$ e $M=\sum_{i=1}^{n} M^{i}$. Além disso, consideramos todos os parâmetros iguais entre as cidades. Para adimensionalizar o modelo definimos as novas variáveis adimensionais independente e dependentes: $\bar{t}=t \nu, \bar{M}_{S}=\frac{M_{S}}{k}, \bar{M}_{I}=\frac{M_{I}}{k}, \bar{H}_{S}=\frac{H_{S}}{H}$ e $\bar{H}_{I}=\frac{H_{I}}{H}$, os quais nos dão um sistema com cinco parâmetros adimensionais: $\bar{\mu}_{H}=\frac{\mu_{H}}{\nu}, \bar{\mu}_{M}=\frac{\mu_{M}}{\nu}, \bar{\beta}=\frac{\beta H}{\nu}, \bar{\lambda}=\frac{\lambda k}{\nu}$ e $\alpha=\frac{\alpha}{\nu}$; obtendo o modelo adimensional e removendo as barras para que notação não fique carregado tem-se 


$$
\left\{\begin{array}{l}
\frac{d M_{S}}{d t}=M(1-M)-\mu_{M} M_{S}-\beta M_{S} H_{I} \\
\frac{d M_{I}}{d t}=-\mu_{M} M_{I}+\beta M_{S} H_{I} \\
\frac{d H_{S}}{d t}=\mu_{H}\left(1-H_{S}\right)-\lambda H_{S} M_{I} \\
\frac{d H_{I}}{d t}=-\left(\mu_{H}+\alpha\right) H_{I}+\lambda H_{S} M_{I} .
\end{array}\right.
$$

As condições iniciais adimensionais $H_{S}(0), M_{S}(0), M_{I}(0), H_{I}(0) \in[0,1]$. Por conseguinte, o espaço de fases é dado por

$$
\Omega=\left\{\left(M_{S}, M_{I}, H_{S}, H_{I}\right) \in \mathbb{R}_{+}^{4} \mid M_{S}+M_{I} \leq 1 \quad \text { e } H_{S}+H_{I} \leq 1\right\} .
$$

O sistema (2) tem três pontos de equilíbrio: $P_{0}=(0,0,1,0), P_{1}=\left(1-\mu_{M}, 0,1,0\right)$, o $P_{1}$ existe se $\mu_{M}<1$, e $P_{2}=\left(M_{S}^{*}, M_{I}^{*}, H_{S}^{*}, H_{I}^{*}\right)$, onde:

$$
\begin{aligned}
H_{I}^{*} & =\frac{\lambda \mu_{H}\left[\lambda \beta\left(1-\mu_{M}\right)-\mu_{M}\left(\mu_{H}+\alpha\right)\right]}{\lambda \beta\left(1-\mu_{M}\right)\left(\mu_{H}+\alpha\right)+\mu_{H} \beta\left(\mu_{H}+\alpha\right)} \\
H_{S}^{*} & =\frac{1}{\mu_{H}}\left\{\mu_{H}-\left(\mu_{H}+\alpha\right) H_{I}^{*}\right\} \\
M_{I}^{*} & =\left\{\frac{1-\mu_{M}}{\mu_{M}+\beta H_{I}^{*}}\right\} \beta H_{I}^{*} \\
M_{S}^{*} & =1-\mu_{M}-M_{I}^{*}
\end{aligned}
$$

para $\mu_{M}<1, H_{I}^{*}<\frac{\mu_{H}}{\mu_{H}+\alpha} \quad$ e $\quad 1<\frac{\lambda \beta\left(1-\mu_{M}\right)}{\mu_{M}\left(\mu_{H}+\alpha\right)}$.

Logo, por meio da matriz da próxima geração [5], obtemos o número de reprodutibilidade basal, $\mathcal{R}_{0}=\sqrt{\frac{\lambda \beta}{\mu_{M}}\left(\frac{1-\mu_{M}}{\mu_{H}+\alpha}\right)}$ que é o raio espectral da matriz da próxima geração,

$$
\mathbb{V}^{-1} \mathbb{F}=\left[\begin{array}{cc}
0 & \frac{\beta}{\mu_{M}}\left(1-\mu_{M}\right) \\
\frac{\lambda}{\mu_{H}+\alpha} & 0
\end{array}\right]
$$

De modo que a estabilidade local é resumida em:

- $\mu_{M}>1 \Longrightarrow$ existe apenas $P_{0}$ e é estável.

- $\mu_{M}<1$ e $\mathcal{R}_{0}<1 \Longrightarrow$ existem $P_{0}$ e $P_{1}$ tais que $P_{0}$ é instável e $P_{1}$ é estável.

- $\mu_{M}<1$ e $\mathcal{R}_{0}>1 \Longrightarrow$ existem $P_{0}, P_{1}$ e $P_{2}$ tais que $P_{0}$ e $P_{1}$ são instáveis, no entanto $P_{2}$ é estável se $a_{1}>0, a_{0}>0$ e $a_{2} a_{1}>a_{0}$, onde

$$
\begin{aligned}
a_{2}= & \mu_{M}+2 \mu_{H}+\alpha+\beta H_{I}^{*}+\lambda M_{I}^{*} \\
a_{1}= & \left(\mu_{M}+\beta H_{I}^{*}\right)\left(\mu_{H}+\alpha\right)+\left(\mu_{M}+\mu_{H}+\alpha+\beta H_{I}^{*}\right)\left(\mu_{H}+\lambda M_{I}^{*}\right) . \\
& -\lambda \beta M_{S}^{*} H_{S}^{*} \\
a_{0}= & \left(\mu_{M}+\beta H_{I}^{*}\right)\left(\mu_{H}+\alpha\right)\left(\mu_{H}+\lambda M_{I}^{*}\right)-\lambda \beta \mu_{H} M_{S}^{*} H_{S}^{*}
\end{aligned}
$$


A Função de Lyapunov para $P_{1}[3]$ é dada por

$$
Q=\frac{\omega_{1}}{\mu_{M}} M_{I}+\frac{\omega_{2}}{\mu_{H}+\alpha} H_{I},
$$

onde $\omega^{T} \geq 0$ é um autovetor à esquerda da matriz $\mathbb{V}^{-1} \mathbb{F}$ correspondente ao autovalor que define $\mathcal{R}_{0}$

De fato,

$$
\begin{aligned}
Q\left(P_{1}\right) & =0 . \\
Q^{\prime}(P) & =\left[H_{S}-\frac{1}{\mathcal{R}_{0}^{2}}\right] \frac{\lambda}{\mu_{H}+\alpha} M_{I}+\left[\frac{M_{S}}{1-\mu_{M}} \mathcal{R}_{0}-1\right] H_{I} \\
& <0, \text { desde que } \mathcal{R}_{0}<1 \text { e } P \neq P_{1} .
\end{aligned}
$$

Portanto, $P_{1}$ é globalmente estável se $\mathcal{R}_{0}<1$.

Também temos o seguinte teorema que garante a invariância do modelo (2) que é um sistema de equações diferenciais. Deste modo o modelo está bem planteado e tem solução no mesmo espaço, consequentemente satisfaz o sentido biológico; a prova do teorema está em Cuba (2020), [2].

Teorema 2.1. O espaço de fases $\Omega$ do modelo (2) é positivamente invariante pelo fluxo.

\section{Simulações}

Para as simulações dividimos o Peru em 5 regiões, Litoral, Interior, Planalto, Serra e Cordilheira, de acordo com a Tabela 1, escolhemos 66 cidades: C1,..,C66, $n=66$, devido ao seu tamanho, importância na região a que pertence e, ou, por ter uma localização importante seja na rede rodoviária ou na rede fluvial (vide a Figura 1). Consideramos o fluxo por rodovias entre as cidades, exceto entre as cidades da região norte da Amazônia peruana, onde só consideramos o fluxo por meio de barcos. O ano de 1990 é considerado como sendo o ano de início da propagação da dengue no Peru. As simulações foram feitas no software GNU Octave por meio de códigos que utilizam o pacote ode23, com os parâmetros da Tabela 1 onde são apresentados os parâmetros utilizados em cada uma das 5 regiões e para todas as regiões consideramos $\alpha=10^{-1}, \mu_{H}=4,0849 \times 10^{-5}, \sigma_{H}=$ $6 \times 10^{-2}$ que é o parâmetro que está ligado ao transporte dos humanos, $\sigma_{M}=10^{-7}$ quando a conexão das cidades é pela rodovia, e $\sigma_{M}=10^{-7}$ quando a conexão das cidades é pela hidrovia, sendo estos dois últimos os parâmetros que estão ligados à migração dos mosquitos.

As condições iniciais em cada cidade a cada novo ano serão definidas dependendo do seu tamanho no ano anterior ao final do período favorável ao mosquito. Consideramos para o $n$ ésimo ano da simulação, $n \geq 2$, a condição inicial, tempo inicial 0 , em cada cidade $i$ dada por $\left(H_{S}^{i}(0, n), H_{I}^{i}(0, n), M_{S}^{i}(0, n), M_{I}^{i}(0, n)\right)$ onde o tempo final $t_{f}=270 \mathrm{e}$

$$
\begin{aligned}
& M_{S}^{i}(0, n)=\left\{\begin{aligned}
\frac{M_{S}^{i}\left(t_{f}, n-1\right)}{10} ; & \text { se } 10^{-4} \leq M_{S}^{i}\left(t_{f}, n-1\right) \leq k^{i} \\
M_{S}^{i}\left(t_{f}, n-1\right) ; & \text { se } 10^{-5} \leq M_{S}^{i}\left(t_{f}, n-1\right)<10^{-4} \\
0 \quad & ; \text { se } 0 \leq M_{S}^{i}\left(t_{f}, n-1\right)<10^{-5},
\end{aligned}\right. \\
& M_{I}^{i}(0, n)=0, \\
& H_{S}^{i}(0, n)=H^{i}-\left(H_{I}^{i}\left(t_{f}, n-1\right)+H_{R}^{i}\left(t_{f}, n-1\right)\right), \\
& H_{I}^{i}(0, n)=\left\{\begin{array}{r}
0,002 ; \text { se } 0,001 \leq H_{I}^{i}\left(t_{f}, n-1\right) \leq H \text { e } M_{S}^{i}\left(t_{f}, n-1\right) \geq 10^{-5} \\
0 ; \text { se } 0 \leq H_{I}^{i}\left(t_{f}, n-1\right)<0,002 \text { e } M_{S}^{i}\left(t_{f}, n-1\right) \geq 10^{-5} .
\end{array}\right.
\end{aligned}
$$


Tabela 1: Parâmetros das cinco regiões consideradas no Peru.

\begin{tabular}{cccccc}
\hline Região & $\beta$ & $\lambda$ & $\nu$ & $\mu_{M}$ & $k$ \\
\hline Litoral & $3,75 \times 10^{-1}$ & $7,5 \times 10^{-1}$ & $10^{-1}$ & $5 \times 10^{-2}$ & $10^{-1}$ \\
Interior & $3,75 \times 10^{-1}$ & $7,5 \times 10^{-1}$ & $10^{-1}$ & $5 \times 10^{-2}$ & $10^{-1}$ \\
Planalto & $2 \times 10^{-1}$ & $5 \times 10^{-1}$ & $1,25 \times 10^{-1}$ & $5 \times 10^{-2}$ & $2 \times 10^{-2}$ \\
Serra & $1,5 \times 10^{-1}$ & $4 \times 10^{-1}$ & $1,42 \times 10^{-1}$ & $5 \times 10^{-2}$ & $10^{-2}$ \\
Cordilheira & 0 & 0 & 0 & $5 \times 10^{-1}$ & $10^{-7}$ \\
\hline
\end{tabular}

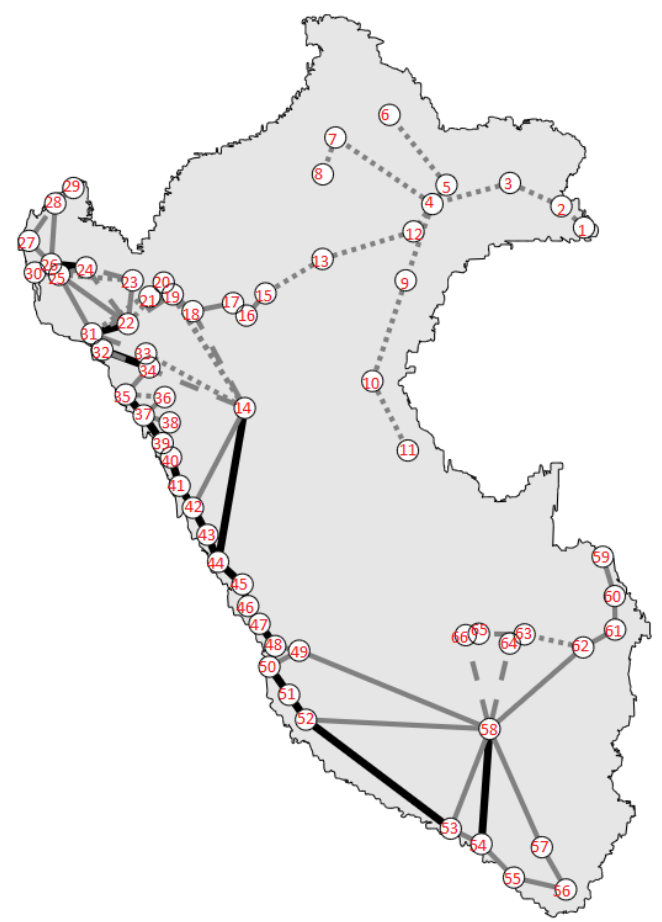

Figura 1: Localização das 66 cidades escolhidas no Peru. As linhas pontilhadas, tracejadas, finas e grosas representam as ligações com seus respectivo fluxo de veículos entre as cidades; quanto mais grosa e obscura é a linha é maior a intensidade do fluxo de veículos entre as cidades. 


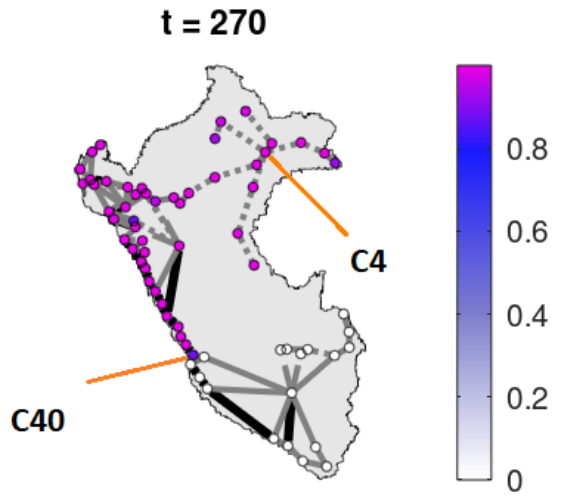

(a) Vigésimo nono ano da propagação da dengue clássica com inicio na região norte na cidade C4.

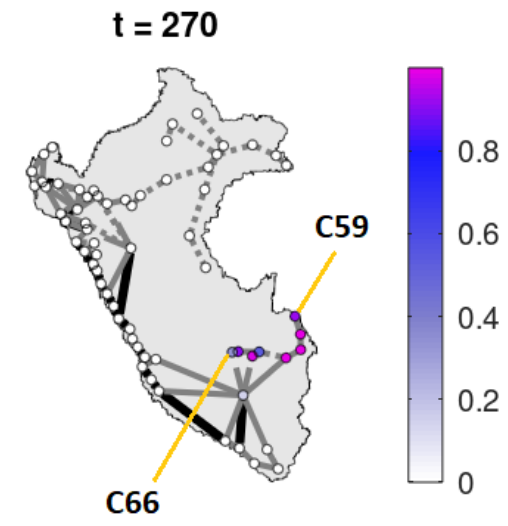

(b) Nono ano da propagação da dengue clássica com inicio na região sul na cidade C59.

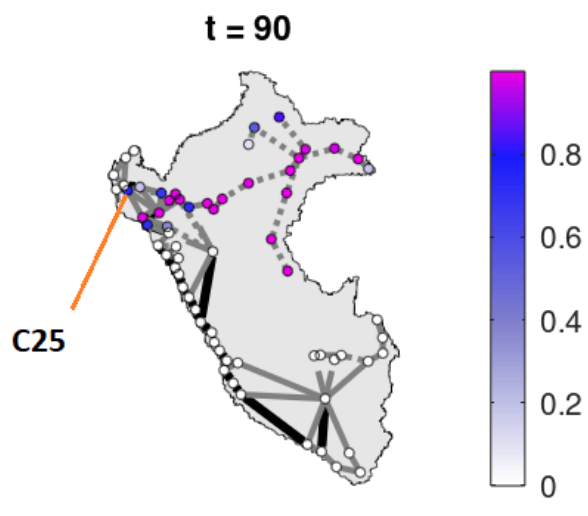

(c) Surto da dengue em 2010 .

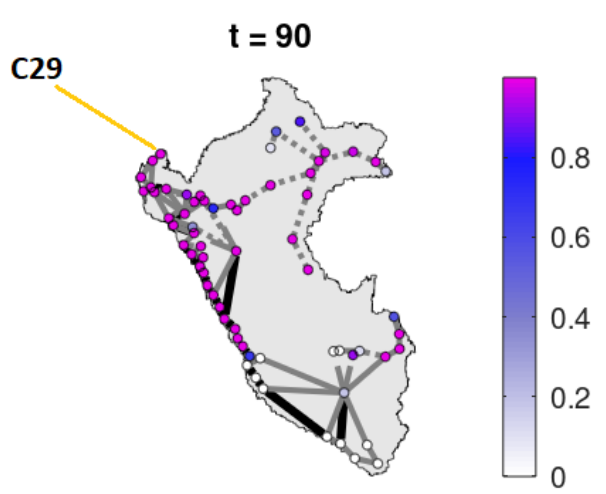

(d) Epidemia da dengue ao ser introduzida um novo sorotipo, DEN-5, em 2020.

Figura 2: População $H_{R}^{i}, i=1, \ldots, 66$, durante durante a propagação da dengue. As barras coloridas ao lado das sub-figuras indicam a proporção dos humanos recuperados.

\subsection{Resultados}

Se a propagação inicia pela região norte da Amazônia peruana na cidade C5-Iquitos em 1990 temos que nos primeiros anos a dengue se espalha pelas principais cidades dessa região, chegando em 11 anos à região norte do Litoral peruano. E em 30 anos só atinge à região norte e centro da Amazônia e do Litoral peruano, vide a Figura 2(a). Além disso, a dengue que chegou a Ayacucho possivelmente não é proveniente da região norte do Peru, pois o primeiro caso da dengue em Ayacucho foi reportada em 2013 na cidade de C66-San Francisco. Porém se a propagação inicia pela região sul da Amazônia peruana na cidade C59-Iñpari em 2007, pois nesse ano iniciou-se a construção da Rodovia Interoceânica que conecta os países do Brasil, Bolívia e Peru aumentando o fluxo de veículos entre estos países, temos que a dengue chega a Ayacucho na cidade C66 no nono 
ano de propagação; a dengue que chega ao Litoral peruano possivelmente não é proveniente do sul do Peru, vide a Figura 2(b). Além disso, a região sul do Peru provavelmente foi contagiada pelo estado do Acre do Brasil.

Se tentamos descrever a propagação geográfica de novos sorotipos, para isto consideremos todos os humanos completamente susceptíveis e mantemos as condições inciais dos mosquitos que já foram espalhadas pelas anteriores simulações, temos acontecimentos similares à realidade com a primeira (2001) e segunda (2010) epidemia da dengue hemorrágica no Peru, vide a Figura 2(c). E a chegada do sorotipo DEN-5 produziria uma epidemia no Peru no verão de 2020, vide a Figura 2(d).

\section{Discussões}

O trabalho teve como objetivo estudar a propagação geográfica da dengue no Peru e descrever o possível caminho percorrido até chegar ao estado do Ayacucho, partindo dos primeiros casos com confirmação laboratorial e iniciando desde o ano 1990 até o 2019. Os resultados obtidos nas simulações são similares aos dados reais do MINSA. E se no verão de 2020 houvesse chegado o DEN-5, possivelmente nesse verão o Peru tivesse passado frente a uma nova epidemia da dengue.

Para descrever essa propagação tivemos que mudar o modelo proposto por Takahashi et al. (2004), pois nesse estudo consideram o mosquito já espalhado. Nosso caso o mosquito no Peru estava iniciando a se espalhar, por tal motivo além de considerar a migração dos mosquitos infectados nós consideramos a migração dos mosquitos suscetíveis, também agregamos o transporte fluvial. Assim, neste trabalho temos além da propagação da dengue o espalhamento do mosquito no território peruano. Além disso, no Takahashi et al. (2004), não foram feitas tanto estudo qualitativo do ponto de equilíbrio endêmico do modelo considerando uma só região e a invariância desse modelo, também o número de reprodutibilidade Basal foi obtido pelo método clássico, nós obtivemos por meio da matriz da próxima geração obtendo o mesmo resultado.

\section{Agradecimentos}

Ao CAPES pelo apoio econômico com o código de financiamento 001 e ao professor Dr. Luis Alberto D'Afonseca pela ajuda com os programas.

\section{Referências}

[1] Cabezas, C., Fiestas, V., García-Mendoza, M., Palomino, M., Mamani, E. e Donaires, F. Dengue en el Perú: a un cuarto de siglo de su reemergencia, Revista peruana de medicina experimental y salud pública, 32:146-156, 2015.

[2] Cuba, N. Q. Um estudo da propagação geográfica da dengue no Peru, Dissertação de Mestrado, UFJF, 2020.

[3] Shuai, Z. e van den Driessche, P. Global stability of infectious disease models using Lyapunov Functions, SIAM Journal on Applied Mathematics, 73:1513-1532, 2013. DOI: https://doi.org/10.1137/120876642.

[4] Takahashi, L. T., Ferreira Jr., W. C. e D’Afonseca, L. A. Propagarão da dengue entre cidades, Biomatemática, 14:1-18, 2004.

[5] van den Driessche, P. e Watmough, J. Reproduction numbers and sub-threshold endemic equilibria for compartmental models of disease transmission, Mathematical biosciences, 180:2948, 2002. DOI:10.1016/S0025-5564(02)00108-6. 